\title{
Análise das prescrições farmacológicas para pacientes em crise hipertensiva em Unidade de Pronto Atendimento de Gurupi - Tocantins
}

\section{Analysis of pharmacological prescriptions for patients with hypertensive crisis in a Gurupi- Tocantins Emergency Care Unit}

${ }^{1}$ Danival Ferreira de Castro, ${ }^{2}$ Lorena Passos Soares, ${ }^{3}$ Wengmo Lima Santos, ${ }^{4}$ João Eduardo Borges Monteiro do Prado, ${ }^{5}$ Andrey Reis da Fonseca, 6 Joelcy Pereira Tavares.

\section{RESUMO}

Introdução: A crise hipertensiva caracteriza-se pela elevação aguda da pressão arterial apresentando risco de lesões em órgãos-alvo e morte, necessitando de rápida intervenção. Objetivo: Traçar o perfil dos pacientes em crise hipertensiva e analisar a conduta farmacológica empregada. Material e Métodos: Estudo retrospectivo, descritivo, realizado na Unidade de Pronto Atendimento de Gurupi-Tocantins, entre abril a setembro de 2017. Foram incluídos na pesquisa todos os prontuários de pacientes atendidos e tratados nesta unidade com diagnóstico de crise entre 2006 a 2016. As variáveis foram sexo, faixa etária, classe medicamentosa e vias de administração. Os dados obtidos foram descritos por meio de coeficiente de correlação de Pearson, regressão linear, médias aritméticas, porcentagens, formas tabulares e gráficos. Realizou-se testes estatísticos por meio do software Analysis of variance (ANOVA), com nível de significância de 5\%. Resultados: Dos 320 pacientes atendidos em crise hipertensiva, houve uma maior prevalência no sexo feminino, na faixa etária entre 51 a 60 anos. Os anti-hipertensivos mais prescritos foram da classe dos inibidores da enzima conversora da angiotensina e dos bloqueadores dos canais de cálcio sendo o captopril e a nifedipina seus principais representantes respectivamente. Observou-se que a via sublingual foi utilizada $19 \%$ das vezes, principalmente no sexo feminino com uso do captopril. Conclusão: A população alvo das crises hipertensivas foram pacientes do sexo feminino, na quinta década de vida. Apesar de proscrita, o uso da via sublingual foi significativo, demonstrando a necessidade de implantação de protocolos e incentivo de adesão aos mesmos pelos profissionais da saúde.

Palavras-chave: Hipertensão. Crise hipertensiva. Administração sublingual.

\section{ABSTRACT}

Introduction: The hypertensive crisis is characterized by an acute elevation of blood pressure presenting high risk of target organ damage and death, necessitating rapid intervention. Objective: Outline the profile of patients in a hypertensive crisis and to analyze the pharmacological management employed. Material and Methods: Retrospective, descriptive study, performed at the Gurupi-Tocantins Emergency Care Unit, between April and September 2017. The study includes all records of patients seen in this unit diagnosed with hypertensive crisis between 2006 and 2016. The variables used were sex, age, drug class and routes of administration. The obtained data were described by means of Pearson correlation coefficient, linear regression, arithmetic means, percentages, tabular forms and graphs. Statistical tests were performed using Analysis of variance (ANOVA) software, with a significance level of $5 \%$. Results: Of the 320 patients treated in a hypertensive crisis, there was a higher prevalence in the female sex, in the age group between 51 and 60 years. The most commonly prescribed antihypertensive agents were in the class of angiotensin converting enzyme inhibitors and calcium channel blockers, with captopril and nifedipine being their main agents, respectively. It was observed that the sublingual route was used $19 \%$ of the time, mainly in females with captopril. Conclusion: The target population of hypertensive crises were female patients in the fifth decade of life. Although banned, the use of the sublingual route was significant, demonstrating the need to implement protocols and encourage adherence to them by health professionals.

Keywords: Hypertension. Hypertensive crisis. Administration Sublingual 


\section{INTRODUÇAOO}

As doenças cardiovasculares representam importante problema de saúde pública no Brasil, sendo responsáveis por mais de 250.000 mortes por ano, incluída nesse grupo está a hipertensão arterial sistêmica (HAS) que acomete cerca de 30\% da população adulta mundial. ${ }^{1}$

De acordo com a $7^{\text {a }}$ Diretriz Brasileira de Hipertensão Arterial, ${ }^{2}$ a HAS é uma condição clínica multifatorial expressa por níveis elevados e sustentados de pressão arterial (PA). Configura-se como precursora de alterações morfofuncionais de órgãos-alvos como o coração, encéfalo, rins e vasos sanguíneos, além de alterações metabólicas, elevando o risco de acidente vascular encefálico, doença arterial coronariana, insuficiência cardíaca, insuficiência renal crônica e doença vascular periférica. ${ }^{3-5}$ Esse quadro de aumento da PA, quando não for devidamente diagnosticado e controlado, pode evoluir para as crises hipertensivas.

A crise hipertensiva é caracterizada por uma elevação aguda e sintomática da PA, com níveis pressão diastólica maior ou igual a $120 \mathrm{mmHg}$, com potencial risco de lesão de órgãos-alvo (LOA) e morte. O controle pressórico em longo prazo é o método mais eficaz para redução de sua incidência. ${ }^{2,6-8}$

Segundo a Sociedade Brasileira de Cardiologia ${ }^{2}$ a crise hipertensiva é dividida em urgências e emergências hipertensivas. Nas urgências, a elevação da PA é superior ao percentil 99 mais $5 \mathrm{mmHg}$ e está associada a sintomas menos lesivos como cefaleia, epistaxe, desmaios e agitação psicomotora e com baixo risco de morte. ${ }^{9}$ Nessa ocasião, o controle da PA pode ser feito por via oral ou, excepcionalmente, sublingual, ${ }^{10-11} \mathrm{com}$ redução da PA em 24 horas.

Já nas emergências hipertensivas a elevação da PA associa-se há sinais que indicam lesão progressiva em órgãos-alvo, constituindo risco imediato à vida do paciente. ${ }^{11-14}$ Os principais sinais são dor torácica, dispneia e déficits neurológicos e estão diretamente relacionados às lesões em órgãos vitais podendo clinicamente se apresentar como infarto agudo do miocárdio, edema agudo de pulmão, encefalopatia hipertensiva e acidente vascular cerebral hemorrágico. ${ }^{9}$ Seu manejo deve ser feito de maneira rápida, com antihipertensivos intravenosos tituláveis em bombas de infusão contínua e monitorização rigorosa da PA. Os protocolos exigem uma redução de 30\% da meta pressórica em 6 a 12 horas, 30\% em 24 horas, com ajuste final em 2 a 4 dias. $^{2}$ 
As crises hipertensivas são eventos frequentes em atendimentos nas unidades de urgência, por isso merece destaque o seu pronto conhecimento, bem como o manejo terapêutico adequado. Além da compreensão clínica da crise hipertensiva, é necessário que o profissional de saúde tenha profundo conhecimento dos anti-hipertensivos bem como a utilização correta das vias de administração.

Além da via oral e endovenosa há outras vias menos comuns de administração de fármacos anti-hipertensivos, porém menos seguras e até mesmo proscritas. Drogas como a nifedipina e o captopril são amplamente utilizadas por via sublingual em unidades de pronto atendimento (UPA); sendo considerada pela Sociedade Brasileira de Cardiologia ${ }^{2}$ uma má prática médica e potencialmente lesiva ao paciente por apresentar efeito errático em sua ação. Seus usos por esta via podem gerar alteração no ritmo cardíaco e a perda de controle na redução da PA, uma vez que reduções muito intensas podem ocasionar acidentes vasculares graves. ${ }^{13}$ Este estudo teve como objetivo analisar o perfil dos pacientes diagnosticados com crise hipertensiva, as principais classes farmacológicas empregadas e suas respectivas vias de administração em uma unidade de pronto atendimento em Gurupi-Tocantins.

\section{MATERIAIS E METODOS}

Foi realizado um estudo do tipo retrospectivo descritivo, transversal e documental. A pesquisa foi realizada na UPA no município de Gurupi, Estado do Tocantins, no período de março a outubro de 2017; sob autorização do Comitê de Ética em Pesquisa com parecer 2.203.607. Foram incluídos na pesquisa todos os prontuários de pacientes atendidos nesta unidade com diagnóstico de crise hipertensiva e tratados com medicamentos antihipertensivos entre 2006 a 2016.

As variáveis de interesse foram: faixa etária, sexo, fármacos utilizados e via de administração medicamentosa.

Os dados obtidos foram analisados por meio de coeficiente de correlação de Pearson, regressão linear, médias aritméticas, porcentagens, além de formas tabulares e gráficos. Realizaram-se testes estatísticos por meio do software Analysis of variance (ANOVA), todos com nível de significância de $5 \%$, ou seja, todas as probabilidades abaixo de 0,05 foram consideradas significativas. 
DOI: 10.18606/2318-1419/amazonia.sci.health.v7n3p62-73 Revista Amazônia: Science \& Health

2019, Vol. 7, № 3

ISSN: 2318-1419
Castro Junior DF. Passos LP. Santos WL. Prado JEBM. Fonseca AR. Tavares JP.

Análise das prescrições farmacológicas para pacientes em crise hipertensi em Unidade de Pronto Atendimento de Gurupi - Tocantins

\section{RESULTADOS}

O estudo abrangeu uma população estatisticamente assimétrica. A média de idade dos pesquisados foi 56 anos, com desvio padrão de 43,13 e coeficiente de variação de 0,76 . Dos 320 pacientes atendidos em crise hipertensiva, foi observada uma maior prevalência da faixa etária entre 51 a 60 anos, com 99 casos (30,9\%). A população feminina foi a mais acometida com $52,8 \%$ dos pacientes (TABELA 1 ).

Tabela 1. Quantidade de pacientes por faixa etária e sexo, atendidos com crise hipertensiva na Unidade de Pronto Atendimento em Gurupi - TO.

\begin{tabular}{cccc}
\hline Faixa etária & Feminino & Masculino & Total Geral \\
\hline 20 a 30 anos & 4 & 7 & 11 \\
31 a 40 anos & 31 & 30 & 61 \\
41 a 50 anos & 19 & 7 & 26 \\
51 a 60 anos & 44 & 55 & 99 \\
61 a 70 anos & 37 & 28 & 65 \\
71 a 80 anos & 24 & 13 & 37 \\
81 a 100 anos & 10 & 11 & 21 \\
Total Geral & 169 & 151 & 320 \\
\hline
\end{tabular}

Ao analisar a quantidade de medicamentos necessários para o controle da crise hipertensiva, foi anotado que a maioria dos pacientes, $57,5 \%$, tiveram os episódios contornados com apenas 1 medicamento. Os casos mais refratários foram dois pacientes, do sexo masculino, que necessitaram de 4 fármacos para resolução do episódio hipertensivo (TABELA 2).

Tabela 2. Número de fármacos anti-hipertensivos por sexo utilizados em pacientes com crise hipertensiva atendidos na Unidade de Pronto Atendimento em Gurupi - TO.

\begin{tabular}{cccccc}
\hline $\begin{array}{c}\text { Quantidade de } \\
\text { medicamentos }\end{array}$ & Feminino & Masculino & Total Geral & $\begin{array}{c}\text { Feminino } \\
(\%)\end{array}$ & $\begin{array}{c}\text { Masculino } \\
(\%)\end{array}$ \\
\hline 1 & 94 & 90 & 184 & $51,1 \%$ & $48,9 \%$ \\
2 & 67 & 49 & 116 & $57,8 \%$ & $42,2 \%$ \\
3 & 8 & 10 & 18 & $44,4 \%$ & $55,6 \%$ \\
4 & 0 & 2 & 2 & $0,0 \%$ & $100,0 \%$ \\
Total & 169 & 151 & 320 & $52,8 \%$ & $47,2 \%$ \\
\hline
\end{tabular}


DOI: 10.18606/2318-1419/amazonia.sci.health.v7n3p62-73 Revista Amazônia: Science \& Health

2019, Vol. 7, № 3 .

ISSN: $2318-1419$
Castro Junior DF. Passos LP. Santos WL. Prado JEBM. Fonseca AR. Tavares JP.

Análise das prescrições farmacológicas para pacientes em crise hipertensi em Unidade de Pronto Atendimento de Gurupi - Tocantins

A via de administração medicamentosa mais utilizada observou-se que a via sublingual foi utilizada 91 vezes (19\%), principalmente no sexo feminino, em associação ou não com outras vias dependendo da gravidade do quadro hipertensivo. A administração oral foi a mais utilizada, representando $60,9 \%$ dos casos. Os pacientes que mais receberam anti-hipertensivos por via sublingual estavam na faixa etária entre 51 a 60 anos, destes 55\% eram do sexo feminino (TABELA 3).

Tabela 3. Uso da via sublingual de acordo com faixa etária e sexo durante o tratamento de crises hipertensivas em pacientes atendidos na Unidade de Pronto Atendimento em Gurupi - TO.

\begin{tabular}{cccccc}
\hline Faixa etária & Masculino & Feminino & Total & Masculino (\%) & Feminino (\%) \\
\hline 20 a 30 anos & 1 & 2 & 3 & $33 \%$ & $67 \%$ \\
31 a 40 anos & 16 & 7 & 23 & $70 \%$ & $30 \%$ \\
41 a 50 anos & 2 & 6 & 8 & $25 \%$ & $75 \%$ \\
51 a 60 anos & 15 & 18 & 33 & $45 \%$ & $55 \%$ \\
61 a 70 anos & 7 & 14 & 21 & $33 \%$ & $67 \%$ \\
71 a 80 anos & 1 & 7 & 8 & $13 \%$ & $88 \%$ \\
81 a 100 anos & 2 & 2 & 4 & $50 \%$ & $50 \%$ \\
Total & 44 & 56 & 100 & $44 \%$ & $56 \%$ \\
\hline
\end{tabular}

Dentre as drogas utilizadas por via sublingual mais prescritas, a classe dos Inibidores da Enzima Conversora da Angiotensina (IECA) foi predominante, sendo o Captopril o seu principal representante em $74,7 \%$ das vezes. Em seguida aparece a classe dos Bloqueadores dos Canais de Cálcio (BCC), na qual a Nifedipina foi a droga mais utilizada (11\%) (TABELA 4).

Tabela 4. Fármacos mais utilizados por via sublingual no controle das crises hipertensivas em pacientes atendidos na Unidade de Pronto Atendimento em Gurupi - TO.

\begin{tabular}{ccc}
\hline Medicamento & $\begin{array}{c}\text { Número de } \\
\text { Utilizações }\end{array}$ & Aplicação (\%) \\
\hline Captopril & 68 & $74,7 \%$ \\
Enalapril & 7 & $7,7 \%$ \\
Furosemida & 1 & $1,1 \%$ \\
Isossorbida & 4 & $4,4 \%$ \\
Nifedipina & 10 & $11,0 \%$ \\
Propanolol & 1 & $1,1 \%$ \\
Total & 91 & $100,0 \%$ \\
\hline
\end{tabular}


DOI: 10.18606/2318-1419/amazonia.sci.health.v7n3p62-73 Revista Amazônia: Science \& Health

2019, Vol. 7, № 3

ISSN: 2318-1419
Castro Junior DF. Passos LP. Santos WL. Prado JEBM. Fonseca AR. Tavares JP.

Análise das prescrições farmacológicas para pacientes em crise hipertensi em Unidade de Pronto Atendimento de Gurupi - Tocantins

A correlação estatística entre a classe medicamentosa e a via utilizada mostrou um valor de $-0,18$ e uma relação $\left(R^{2}\right)$ de 0,0324 , portanto, uma relação fraca e pouco significativa, demostrando que as prescrições não eram protocolares e de indicação pessoal dos prescritores.

Ao analisar as 68 aplicações de captopril via sublingual por faixa etária foi encontrado uma maior prevalência (37\%) das aplicações entre 51 a 60 anos (Gráfico 1).

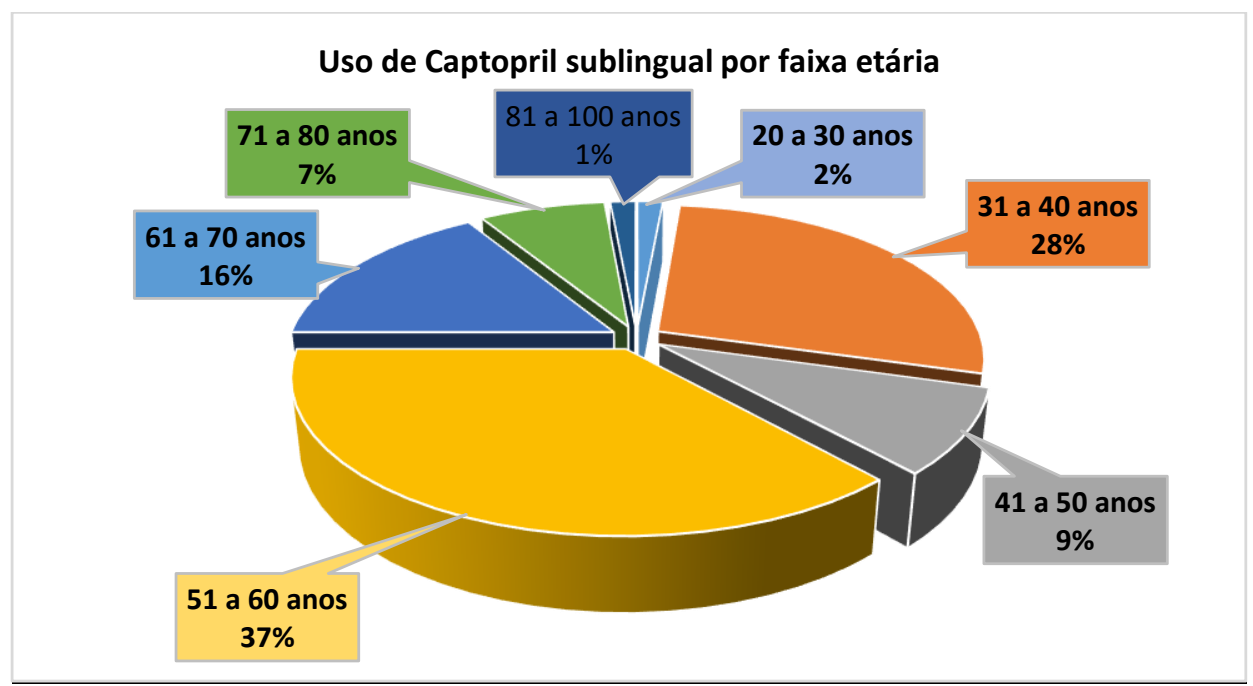

Figura 1. Uso do captopril sublingual por faixa etária no controle das crises hipertensivas em pacientes atendidos na Unidade de Pronto Atendimento em Gurupi - TO.

Em relação ao uso de Nifedipina sublingual por faixa etária, a pesquisa apontou que em $40 \%$ das vezes ela foi prescrita na faixa de 31 a 40 anos (Gráfico 2)

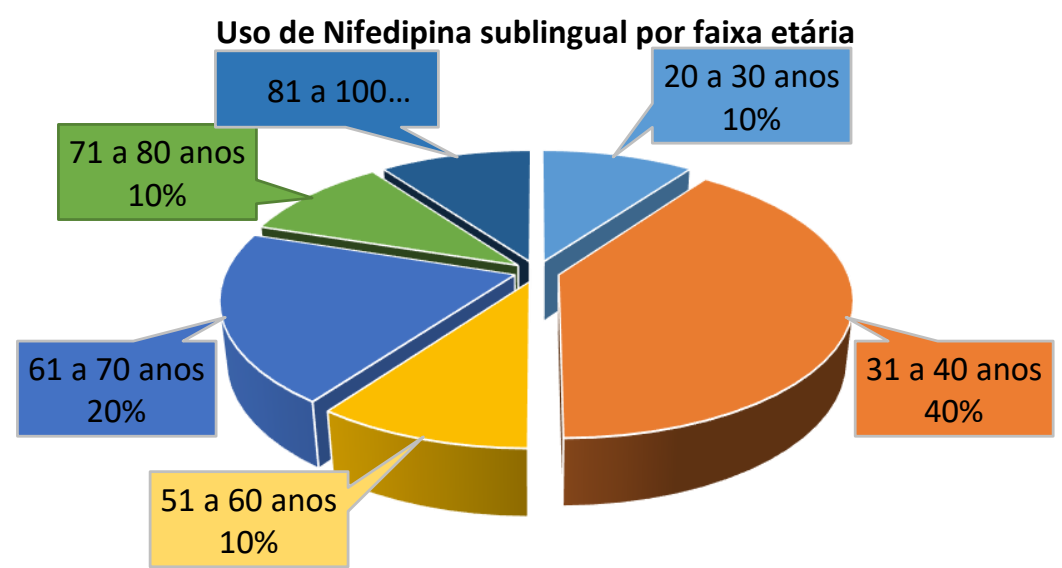


DOI: 10.18606/2318-1419/amazonia.sci.health.v7n3p62-73 Revista Amazônia: Science \& Health
Castro Junior DF. Passos LP. Santos WL. Prado JEBM. Fonseca AR. Tavares JP.

Análise das prescrições farmacológicas para pacientes em crise hipertensi em Unidade de Pronto Atendimento de Gurupi - Tocantins

Figura 2. Uso da Nifedipina sublingual por faixa etária no controle das crises hipertensivas em pacientes atendidos na Unidade de Pronto Atendimento em Gurupi - TO. O uso de captopril e nifedipina por via sublingual foram realizados em pacientes previamente portadores de HAS em 76,47\% e 70\% das vezes, respectivamente.

\section{DISCUSSÁO}

O presente estudo evidencia uma maior prevalência de pacientes em crise hipertensiva que buscam o atendimento de emergência estão numa faixa etária entre 51 a 60 anos, principalmente do sexo feminino. Outros estudos apresentaram achados semelhantes, mesmo com metodologias e amostras populacionais distintas. Num estudo com 557 pacientes Riege ${ }^{14}$ encontrou uma maior taxa de pacientes em crise hipertensiva na faixa etária entre 50 e $59(24,8 \%)$ e foi identificado maior prevalência destes episódios na população feminina (62,5\%). Assim como Pereira ${ }^{13}$ em seu estudo com 107 pacientes com quadros de crise hipertensiva em uma UPA, dos quais 54,2\% eram do sexo feminino.

A prevalência de doenças cardiovasculares cresce com o incremento da idade, especialmente no sexo feminino segundo a Sociedade Brasileira de Cardiologia e a Associação Brasileira de Climatério. ${ }^{15} \mathrm{O}$ estrogênio parece ter efeito protetor no controle da pressão arterial, com o desenvolvimento da falência ovariana os episódios de crises hipertensivas tornam-se mais comuns, uma vez que há a perda de fatores endoteliais protetores mediados pelo estrogênio. ${ }^{16-19}$

O estudo mostrou que o sexo masculino é mais refratário às intervenções farmacológicas, necessitando de maior número de drogas anti-hipertensivas para o controle das crises. Na Revista da Sociedade Brasileira de Hipertensão De Carvalho ${ }^{20}$, descreve alguns fatores associados à refratariedade do controle pressórico nas crises hipertensivas como: técnica inadequada da aferição da PA, comorbidades crônicas prévias e presença de hipertensão secundária. Pacientes do sexo masculino apresentam maior número de doenças multiarteriais o que dificulta o manejo hipertensivo. ${ }^{21}$ A prevalência de hipertensão no sexo feminino é maior, porém os homens são mais displicentes quanto à terapêutica prescrita pelos médicos o que agrava o quadro hipertensivo, ao contrário das mulheres se mostram mais preocupadas com a saúde e têm maior adesão ao tratamento. ${ }^{22-25}$

A via sublingual aparece sendo utilizada em 19\% dos casos na população pesquisada, 
tal achado se mostra preocupante por se tratar de uma via proscrita para intervenção terapêutica nestes casos. Tanto o uso de drogas da classe dos IECAs, como o captopril, quanto da classe dos BCC, como a nifedipina, podem apresentar efeitos erráticos quando usados por via sublingual, uma vez que são capazes de apresentar resposta inapropriada do ritmo cardíaco, no grau de redução da PA e em alguns casos, quando muito intensa, essa resposta pode ocasionar lesões vasculares encefálicas e coronarianas. ${ }^{26}$ Já Oigman ${ }^{25}$ defende que mesmo proscrito, o uso do captopril por via sublingual é seguro por não reduzir tão abruptamente a PA e consequentemente menos efeitos colaterais graves.

Num estudo pioneiro sobre os efeitos da hipertensão arterial crônica sobre a microcirculação sublingual, Kanoore ${ }^{27}$, observou a presença de alterações vasculares em relação à perfusão desta região, o que pode comprometer a absorção de drogas antihipertensivas.

Dentre as prescrições sublinguais observamos uma alta prevalência no uso do captopril por esta via. Um achado semelhante foi obtido por Monteiro ${ }^{28}$ em seu trabalho com 169 pacientes, dos quais 112 utilizaram IECA por via sublingual para o manejo pressórico nas crises hipertensivas.

O captopril é considerado a droga de uso não parenteral mais segura nas situações em que a elevação da PA não está associada à lesão aguda de órgão-alvo e por isso é recomendado pelas diretrizes vigentes. ${ }^{2}$ Mas há discussões discordantes em relação ao seu uso por via sublingual. Alguns trabalhos afirmam que não há vantagens terapêuticas do uso desta via em relação a via oral, ${ }^{29-30}$ outros reforçam que este fármaco perde propriedades farmacocinéticas de absorção quando usado de forma sublingual o que pode diminuir seu efeito. ${ }^{3,6}$

Apesar de a nifedipina representar apenas $11 \%$ das prescrições sublinguais, trata-se de um número alarmante visto que seu uso é considerado como má pratica médica. A $7^{0}$ Diretriz Brasileira de Hipertensão Arterial, ${ }^{2}$ é incisiva em contra-indicar o uso deste fármaco por esta via, seja para o tratamento ambulatorial de HAS ou nas crises hipertensivas. A normativa afirma que há a possibilidade de uma redução acentuada na PA, podendo provocar isquemia tecidual em órgãos nobres como cérebro e tecido cardíaco, mostrando não ser segura quando usada por via sublingual.

Em um estudo no município de Gurupi-TO, NJ Tenório Filho ${ }^{31}$ avaliou efetividade na prescrição anti-hipertensiva relacionada ao controle pressórico em regime ambulatorial no 
DOI: 10.18606/2318-1419/amazonia.sci.health.v7n3p62-73 Revista Amazônia: Science \& Health

2019, Vol. 7, № 3

ISSN: 2318-1419
Castro Junior DF. Passos LP. Santos WL. Prado JEBM. Fonseca AR. Tavares JP.

Análise das prescrições farmacológicas para pacientes em crise hipertensi em Unidade de Pronto Atendimento de Gurupi - Tocantins

município de Gurupi entre 2011 a 2016 e constatou que dos 67 pacientes hipertensos pesquisados, houve predomínio do sexo feminino (52,2\%), e apenas 21 pacientes atigiram as metas pressóricas preconizadas pela $7^{0}$ diretriz brasileira de hipertensão arterial; o que reforça o achado de pior adesão ao tratamento e, consequentemente, maiores índices de agudizações pressóricas por parte do sexo feminino nesta população.

A falta de adesão ao tratamento anti-hipertensivo, utilização de subdoses, presença de comorbidades prévias como doença renal crônica e diabetes melitos colaboram para a alta frequência de crises hipertensivas. ${ }^{5}$

\section{CONSIDERACCOES FINAIS}

Infelizmente, as emergências e urgências hipertensivas estão entre os problemas médicos pouco compreendidos e mal administrados nas unidades de pronto atendimento. No estudo foi possível delinear como população alvo das crises hipertensivas os pacientes do sexo feminino, na faixa etária entre 51 a 60 anos. Verificou-se ainda que apesar da via oral ter sido a mais utilizada, a via sublingual teve uma grande representatividade no tratamento das crises hipertensivas, com destaque para o uso dos inibidores da enzima conversora de angiotensina. A alta incidência do uso da via sublingual, assim como a prática de condutas consideradas proscritas, demonstram o despreparo médico na condução da crise hipertensiva. Fica evidente a real necessidade da implementação de protocolos e atualizações das equipes de pronto atendimento no manejo desta grave condição clínica. Por se tratar de um estudo retrospectivo, atrelado apenas a dados contidos em prontuários, são necessários novos trabalhos a nível regional que corroborem para o delineamento das condutas frente a crises hipertensivas.

\section{REFERENCIAS}

1. Hajjar I, Kotchen TA. Trends in prevalence, awareness, treatment, and control of hypertension in the United States, 1988-2000. JAMA. 2003;290(2):199-206.

2.Malachias MVB, Souza WKSB, Plavnik FL, Rodrigues CIS, Brandão AA, Neves MFT, et al. $7^{a}$ Diretriz Brasileira de Hipertensão Arterial. Arq Bras Cardiol. 2016;107(3):1-83. 
DOI: 10.18606/2318-1419/amazonia.sci.health.v7n3p62-73 Revista Amazônia: Science \& Health

2019, Vol. 7, № 3 .

ISSN: 2318-1419
Castro Junior DF. Passos LP. Santos WL. Prado JEBM. Fonseca AR. Tavares JP.

Análise das prescrições farmacológicas para pacientes em crise hipertens em Unidade de Pronto Atendimento de Gurupi - Tocantins

3.Carnelosso ML, Barbosa MA, Sousa ALL, Monego ET, Carvalho MM. Enfermidades nãotransmissíveis na Atenção Básica: novo desafio para o PSF. Projeto de Desenvolvimento de Sistemas e Serviços de Saúde. 2004;492-501.

4.Malta DC, Moura L, Souza FM, Rocha FM, Fernandes FM. Doenças crônicas não transmissíveis: mortalidade e fatores de risco no Brasil, 1990 a 2006. Saúde Bras. 2008;20:337-62.

5.De Castro Júnior DF, Soares LP, Barbosa RP, Tenório Filho NJ, Vasconcelos GG, de Bessa NF, Herrera SDSC. Prevalência de hipertensão arterial sistêmica e diabetes melitus em pacientes com doença renal crônica em ambulatório de cardiologia. Rev Cereus. 2017;9(3):2-20.

6.Calhoun DA, Oparil S. Treatment of hypertensive crisis. $\mathrm{N}$ Engl $\mathrm{J}$ Med. 1990;323(17):1177-1183.

7.Martin JFV, Higashiama E, Garcia E, Luizon MR, Cipullo JP. Perfil da crise hipertensiva: prevalência e apresentação clínica. Arq Bras Cardiol. 2004;83(3):1-14.

8.Lopes RP, Feitosa Filho GS. Crise hipertensiva. Rev Soc Bras Clín Méd. 2005;3(4):113116.

9.Papadopoulos DP, Mourouzis I, Thomopoulos C, Makris T, Papademetriou V. Hypertension crisis. Blood press. 2010;19(6):328-336.

10.Blumenfeld JD, Laragh JH. Management of hypertensive crises: The scientific basis for treatment decisions. Am J Hypertens. 2001;14(11):1154-67.

11.Nobre F, Chauchar F, Viana JM, Pereira GJ, Lima NK. Evaluation of the medical care of patients with hypertension in an emergency department and in ambulatory hypertension unit. Arq Bras Cardiol. 2002;78(2):156-61.

12.Kaplan N.M. Clinical Hypertension. $7^{\text {a }}$ ed. Baltimore: Williams and Wilkins;1998.

13.Pereira SE, Aguiar RS, Vian VH, Cunha EM, Silva DAD, Teixeira MA. Análise crítica do atendimento da crise hipertensiva em Unidade de Pronto Atendimento de Itaperuna-Rio de Janeiro. Rev Bras Med. 2012;69(5):139-44.

14.Riegel F, Siqueira DS, Tavares JP. Perfil dos pacientes com crise hipertensiva atendidos em um Hospital de Pronto Socorro de Porto Alegre-RS. Clin Biomed Res. 2014; 34(Supl.):142.

15.Sociedade Brasileira de Cardiologia e Associação Brasileira de Climatério. I Diretriz Brasileira sobre Prevenção de Doenças Cardiovasculares em Mulheres Climatéricas e a Influência da Terapia de Reposição Hormonal (TRH) da Sociedade Brasileira de Cardiologia (SBC) e da Associação Brasileira do Climatério (SOBRAC). Arq Bras Cardiol. 2008;1(1). 
DOI: 10.18606/2318-1419/amazonia.sci.health.v7n3p62-73 Revista Amazônia: Science \& Health

2019, Vol. 7, № 3 .

ISSN: 2318-1419
Castro Junior DF. Passos LP. Santos WL. Prado JEBM. Fonseca AR. Tavares JP.

Análise das prescrições farmacológicas para pacientes em crise hipertens em Unidade de Pronto Atendimento de Gurupi - Tocantins

16.Fonseca AM, Bagnoli VR, Arie WMY. A Dúvida do ginecologista: prescrever ou não hormônios na mulher no climatério. Rev Assoc Med Bras. 2009;55(5):507.

17.Lorenzi DRS, Catan LB, Moreira K, Ártico GR. Assistência à mulher climatérica: novos paradigmas. Rev Bras Enferm. 2009;62(2):287-96.

18.Santos ZMSA, Silva RM, Monteiro, DA. Mulher com hipertensão e a relação com a menopausa. Rev RENE. 2006;7(1):68-74.

19.Board of the International Menopause Society, Pines A, Sturdee DW, Birkhäuser MH, Schneider HP, Gambacciani M, Panay N. IMS updated recommendations on postmenopausal hormone therapy. Climacteric. 2007;10(3):181-194.

20.De Carvalho MHC. Hipertensão Arterial Refratária um problema em busca de solução. Rev Bras Hipertens. 2005;8(2):2.

21.Oliveira JLM, Hirata M, Souza AGM. Sexo masculino e hipertensão arterial são preditores de placa à angiotomografia das coronárias. Arq Bras Cardiol. 2015;104(5):409416.

22.Barros MBDA, Francisco PMSB, Zanchetta LM, César CLG. Tendências das desigualdades sociais e demográficas na prevalência de doenças crônicas no Brasil, PNAD: 2003-2008. Ciênc Saúde Colet. 2011;16:3755-3768.

23.Mengue SS, Bertoldi AD, Ramos LR, Farias MR, Oliveira MA, Tavares NUL, et al. Acesso e uso de medicamentos para hipertensão arterial no Brasil. Rev Saúde Pública. 2016;50(suppl 2):1-8s.

24.Gomes R, Moreira MCN, Nascimento EFD, Rebello LEFDS, Couto MT, Schraiber LB. Os homens não vêm! Ausência e/ou invisibilidade masculina na atenção primária. Ciênc Saúde Colet. 2011;16:983-992.

25.Oigman W. Artigo de Revisão Crise hipertensiva: quando e como abordá-la. Rev. Socerj. 2003;1(16):52-59.

26.Almeida FA. Emergências hipertensivas: bases fisiopatológicas para o tratamento. Rev Bras de Hipertens. 2002;9(4):346-352.

27.Kanoore Edul VS, Ince C, Estenssoro E, Ferrara G, Arzani Y, Salvatori C, et al. The effects of arterial hypertension and age on the sublingual microcirculation of healthy volunteers and outpatients with cardiovascular risk factors. Microcirculation. 2015;22(6):485-492.

28.Júnior FDCM, Anunciação FAC, Salgado Filho N, Mayara G, da Silva A, Barbosa JB, et al. Prevalência de verdadeiras crises hipertensivas e adequação da conduta médica em pacientes atendidos em um pronto-socorro geral com pressão arterial elevada. Arq Bras Cardiol. 2008;90(4):269-273. 
29.Lacy C. Drug Information Handbook: A Comprehensive Resource for All Clinicians and Healthcare Professionals. 22aㅡ ed. Ohio: Lexi-Comp Inc; 2013.

30.Mcevoy GK (Ed.). AHFS Drug Information. 1aㅡ ed. Bethesda: ASPH; 2011.

31.Tenório Filho NJ, Largas RO, Oliveira LPS, Silva DDS, Vasconcelos GG, Soares LP, et al. Prescrição Anti-Hipertensiva e Efetividade do Controle Pressórico Ambulatorial. Rev Cereus. 2018;10(3):50-61. 